\title{
ELECTROENCEPHALOGRAPHIC AND CLINICO-PATHOLOGICAL OBSERVATIONS IN HYDROCEPHALIC CHILDREN
}

\author{
BY \\ G. PAMPIGLIONE AND K. M. LAURENCE \\ From The Hospital for Sick Children, Great Ormond Street, London
}

(RECEIVED FOR PUBLICATION MAY 10, 1962)

Hydrocephalus in children is common, and considerable numbers of papers have been published on its clinical, therapeutic and pathological aspects. Following a variety of aetiologies, the encephalon eventually becomes distorted, and the cerebral circulation may be altered by the frequent increase in intracranial pressure as well as by the anatomical changes.

The study of the electrical activity of the brain (E.E.G.) has become a routine procedure in many neurological and neurosurgical centres. However, reports of systematic E.E.G. studies of young hydrocephalic patients have not appeared, and in their book on electroencephalography, Hill and Parr (1950) seem to neglect this condition. The few papers on electroencephalography in hydrocephalus report on small groups of cases, and often lack documentation as to the type of hydrocephalus, its possible aetiology, and concomitant impairment of cerebral function (see Bibliography in Cavazzuti, Canossi and Bergonzini, 1957, and Melchior, 1961).

In the present investigations the electrical activity of grossly distorted brains was studied, together with the results of other investigations, in an attempt to assess the aetiology of the condition in each case, and the possible contribution of neurophysiological studies to this problem.
Material and Methods

Out of 102 patients with hydrocephalus referred in three years (1957-1959) to the Department of Clinical Neurophysiology of The Hospital for Sick Children, London, 50 patients were selected who fulfilled the following criteria. Each child had been admitted to the Hospital and a careful history was taken with special reference to the events at birth, the occurrence of seizures, the rapidity and degree of the enlargement of the head, the state of vision, and the motor and mental development. In addition to the clinical examination, various investigations were performed including phenol-red excretion tests (Laurence, 1957, 1959) and air encephalography, to determine the site of the block, the thickness of the pallium, the presence of cerebral malformations and other gross lesions in each case.

Ventricular pressure readings were taken on most patients on several occasions. All but seven of the children were below the age of 3 years at the time of their first E.E.G. (33 between 1 week to 1 year; 14 between 1 and 4 years; 3 above 4 years of age).

The probable aetiological factors could be deduced in all but five of the 50 cases (Table), and confirmation was obtained in eight patients where post-mortem examination was carried out. In the five cases with obscure aetiology an inflammatory

TABLE

PROBABLE AETIOLOGICAL FACTORS IN HYDROCEPHALIC CHILDREN

\begin{tabular}{|c|c|c|c|c|c|c|c|}
\hline Anatomical Site & of Block & Trauma & Infection & Malformation & Tumours & Unknown & Total \\
\hline Basal cysterns & .. & 16 & $2(+2)$ & 0 & 0 & 5 & 23 \\
\hline Posterior fossa & . & (1) & (5) & 17 & 0 & 0 & 17 \\
\hline Aqueduct $\ldots$ & . & 2 & 3 & 3 & 2 & 0 & 10 \\
\hline Total & .. & $18(1)$ & $5(7)$ & 20 & 2 & 5 & 50 \\
\hline
\end{tabular}

The cases in brackets had multiple aetiology. 
process had been present but it was not possible to say whether this followed trauma or an infection (including three patients with post-mortem examination). Multiple aetiological factors were recognized in eight patients.

Eight patients were studied after a 'Forrest disc' operation (Forrest, Laurence and Macnab, 1957). In four of them records had also been taken before the operation. In 15 patients E.E.G. studies were performed after the condition had become naturally arrested, while in five others the E.E.G.s were taken during the terminal phase of the illness.

All the records were carried out with an eightchannel apparatus (Offner Type D 111) with an amplification of 10 microvolts per millimetre pen deflection, although the gain often had to be reduced. The H.F. response was linear within $10 \%$ to $60 \mathrm{c} / \mathrm{s}$ (decreasing gradually thereafter). The time constant was always of 0.35 seconds with the exception of a few cases when a D.C. coupler was employed in either one or two channels. The electrodes were silver-silver chloride discs stuck to the scalp with collodion, the contact resistance being reduced to below $3 \mathrm{kOhms}$ by a minimal abrasion of the epidermis and a saline jelly. Often 20 electrodes were used, but a minimum of 12 was employed in very ill babies. In two patients records were taken during slow withdrawal of C.S.F. and two needles were inserted at the lateral margin of the wide anterior fontanelle, one for continuous monitoring of pressure changes in the C.S.F. and the other for the withdrawal of fluid. A strain gauge was also applied across the fontanelle in order to record pulsation of the brain and changes in tension in relation to respiration, straining or crying of the babies. On six occasions short-acting barbiturates were administered, but the majority of patients had records taken without sedation. Spontaneous sleep often occurred during the test. Particular attention was devoted to the effect of sensory stimuli, including rhythmic photic stimulation.

\section{Results}

Anatomical Aspects. There was no constant correlation between the size of the patient's head, the thickness or thinness of the pallium (as judged by pneumoencephalographic studies) and any particular E.E.G. pattern, except that the E.E.G. was of very low amplitude over completely atrophic cortex and over large collections of subdural fluid, as eventually demonstrated on autopsy. Activity of 100-200 microvolts could still be recorded between electrodes $6-7 \mathrm{~cm}$. apart over a cerebrum reduced to a thickness of only a few millimetres. The following case illustrates this point.

Case L.G.B. After an apparently normal pregnancy and delivery, this child had a normal development until the age of 4 months, when it was noticed that her head was getting large. When she was admitted at the age of 8 months with progressive hydrocephalus, her skull circumference was $55 \mathrm{~cm}$. The E.E.G. (Fig. 1), showed only a mild diffuse abnormality without focal or paroxys-

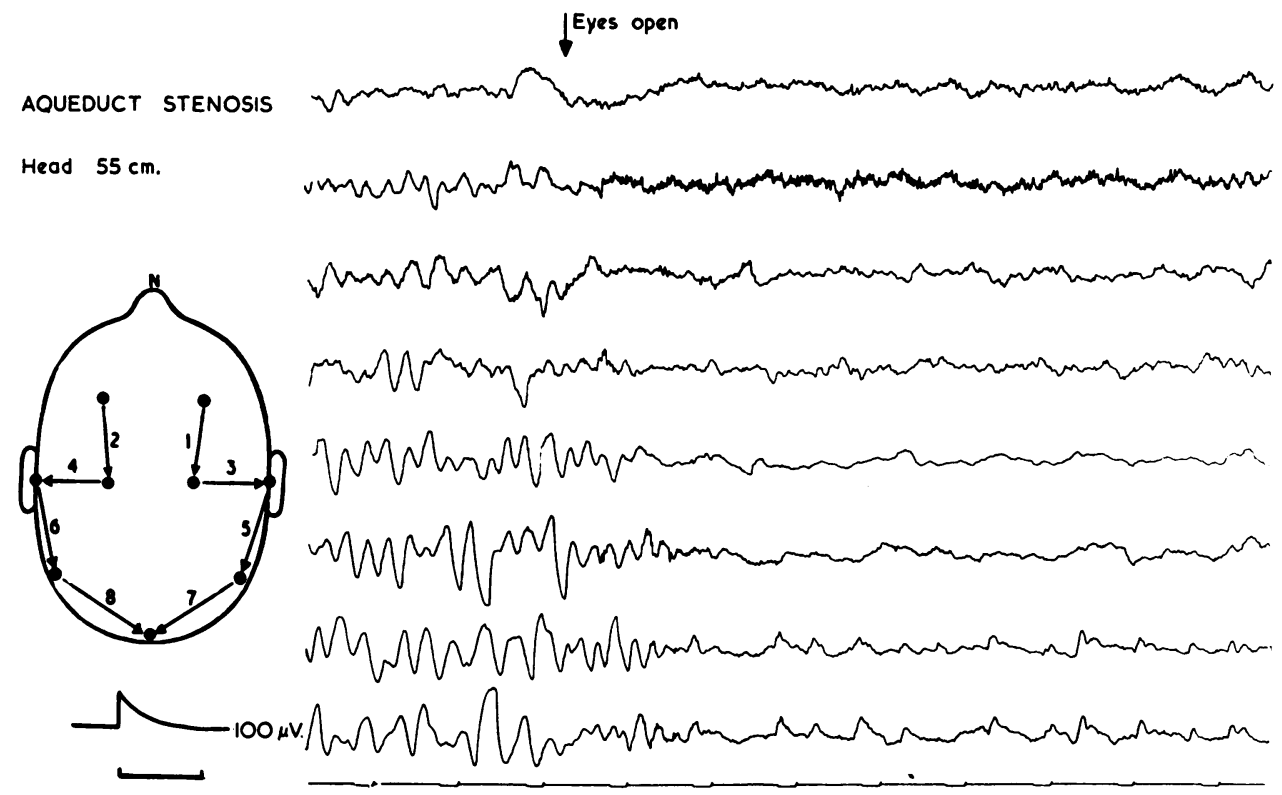

Fig. 1.-L.G.B., age 8 months (head $55 \mathrm{~cm}$.). Aqueduct stenosis. 
mal features and without gross asymmetry in the activity of the two hemispheres. There were considerable fluctuations in the amplitude of 3-4 c/s activity throughout the test, and lambda waves appeared when the baby had her eyes open. The intraventricular pressure was about $300 \mathrm{~mm}$. of C.S.F.

Air ventriculography demonstrated a gross hydrocephalus (pallium less than $1 \mathrm{~cm}$. thick), due to an aqueduct block. A normal subarachnoid space was shown on lumbar air encephalography. A Torkildsen operation and a subsequent Forrest disc operation did not help the patient, who died a few weeks later at the age of 10 months. At autopsy the gross hydrocephalus was found to be due to a gliosis of the aqueduct. Both the Forrest disc and the Torkildsen tube were blocked by dense fibrin deposits. Most of the pallium was about half a centimetre thick or less (Fig. 2).

Clinical Aspects. Several of the patients in whom the condition had become static showed only a mild diffuse E.E.G. abnormality. In rapidly progressing cases the E.E.G. abnormality was sometimes slightly more severe than in the static cases on serial studies. The severity of the increase in the intracranial pressure, however, did not appear to be accompanied by a parallel change in the E.E.G. abnormality.

Case T.H. This baby was born with a large myelomeningocele which was operated upon when the baby was 4 days old (August 28, 1957). Progressive enlargement of the head was noticed in the next few weeks. When he was 5 weeks old the E.E.G. showed only a minimal abnormality without lateralization (Fig. 3), while the responses to photic stimulation were regular and symmetrical. Ventriculography four days after the E.E.G. showed a marked symmetrical hydrocephalus, and an Arnold-Chiari malformation. The pallium was about 2 to $3 \mathrm{~cm}$. thick, and the intraventricular pressure was $180 \mathrm{~mm}$. of C.S.F. A Forrest disc operation was performed on October 14,1957, followed by temporary improvement. A few weeks later, however, the enlargement of the head became rapidly progressive. An E.E.G. taken on February 19, 1958, showed a considerable amount of rhythmic 3-4 c/s activity and some slower waves, slightly more obvious over the left than the right temporo-occipital region. The most striking feature, however, was the increase in amplitude, the activity on occasions being of the order of $\frac{1}{2}$ to 1 millivolt (Fig. 4). In spite of the insertion of the disc in the right hemisphere, only a mild asymmetry was seen between the activity of the two hemispheres and no discharges appeared. At the time the head circumference was $67 \mathrm{~cm}$.; the skull was extremely thin with an enormous confluence of the fontanelles. The large amplitude of the traces was probably related to the close proximity of the electrodes to the cortex without much intervening skull.

No more E.E.G.s were taken on this baby because of the thinness of the coverage of the brain. Three months later the child died, following spontaneous rupture of the skin and of the brain with gushing of C.S.F. At

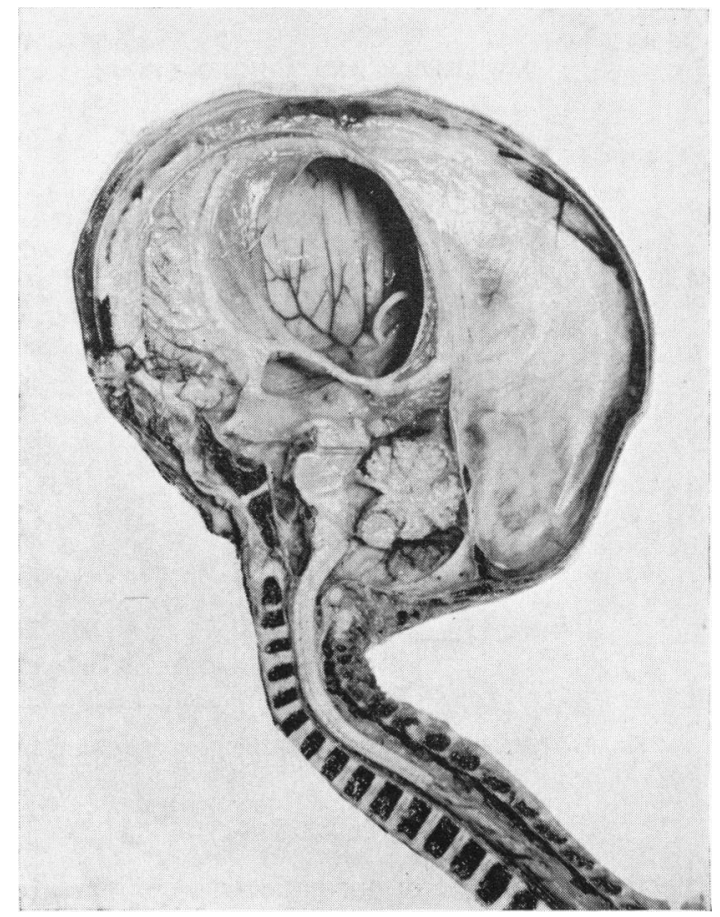

FIG. 2.-L.G.B., gross dilatation of III and both lateral ventricles with very thin pallium. The Torkildsen tube blocked by adhesions sticks out in a loop in the lateral ventricle. Part of the upper brain stem with the stenosis of the aqueduct has been cut away for histological examination.

autopsy, in addition to the ruptured internal hydrocephalus, the Arnold-Chiari malformation and the recent subdural and subarachnoid haemorrhage, there was some necrosis of the cerebral cortex and a cyst of the septum pellucidum.

Seizures. Seizures did not appear to be common in this group of hydrocephalic children, although reliable information was not easy to obtain. Only three of the 50 patients had a definite history of seizures, while another one was said to have had apnoeic attacks in the first week of life. This number is comparable with the experience of other workers (Fois, Gibbs and Gibbs, 1958). Discharges in the E.E.G. in the form of sharp elements or spikes were seen in the four patients with a history of attacks, as well as in six others who never had fits. In all cases the discharges were isolated rather than in groups, and focal rather than generalized. Such discharges were more common in patients where the hydrocephalus was due to a basal cisternal block or to an aqueduct stenosis than in patients with an Arnold-Chiari malformation. It was difficult to appreciate in this connexion the possible effects of various therapeutic attempts and the possible results of the occasional superimposed 
MYELOCELE AND ARNOLD-CHIARI

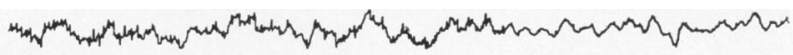

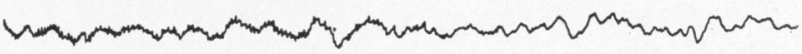

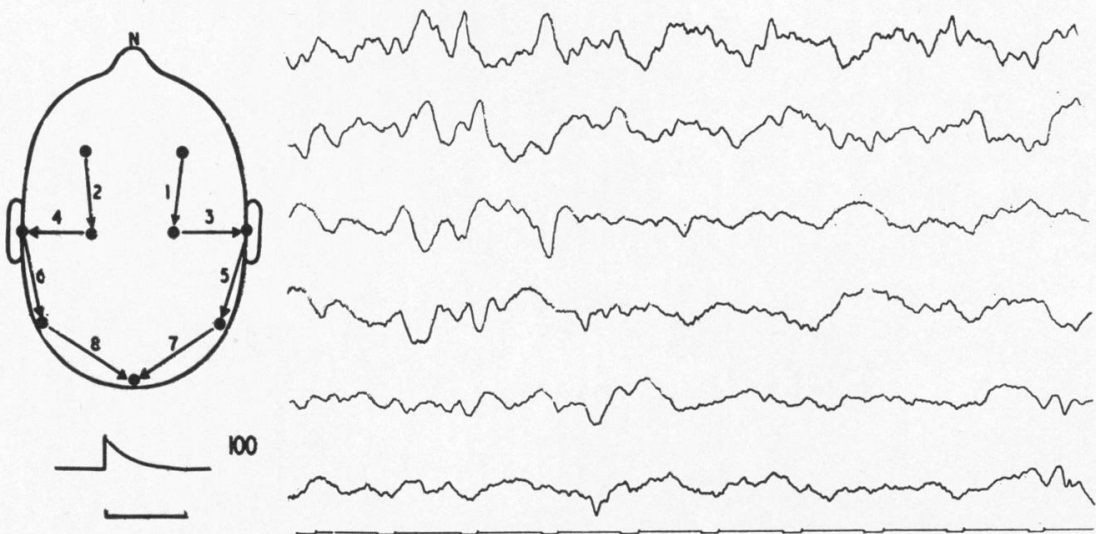

FIG. 3.-T.H. age 5 weeks. E.E.G. on September 30, 1957; diagnosis-myelocele and Arnold Chiari. Head measured $40 \mathrm{~cm}$.

infections. In none of our patients, were repetitive complex discharges (such as spike and wave complexes) seen either spontaneously or during photic stimulation.
Case S.R. This girl was born with a large head, and there was some motor and mental retardation in her early development. At the age of 2 years her head circumference was $65 \mathrm{~cm}$. She was unable to stand
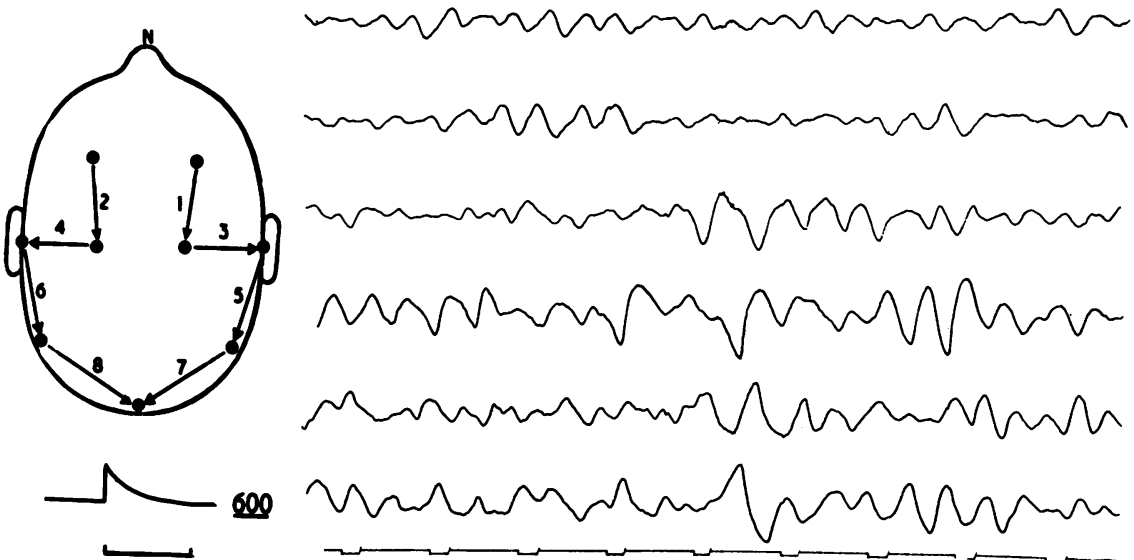

600
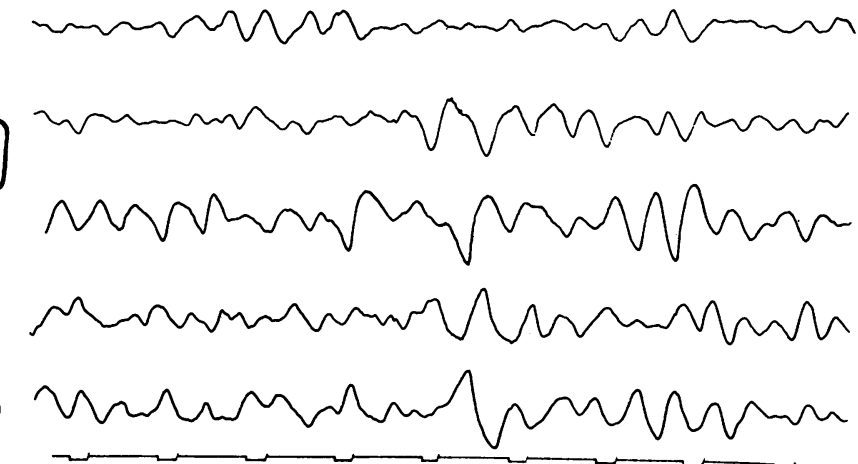

Fig. 4.-T.H., age 6 months, E.E.G. recorded on February 19, 1958. Very thin cortex Head $67 \mathrm{~cm}$. Disc operation October $14,1957$. 


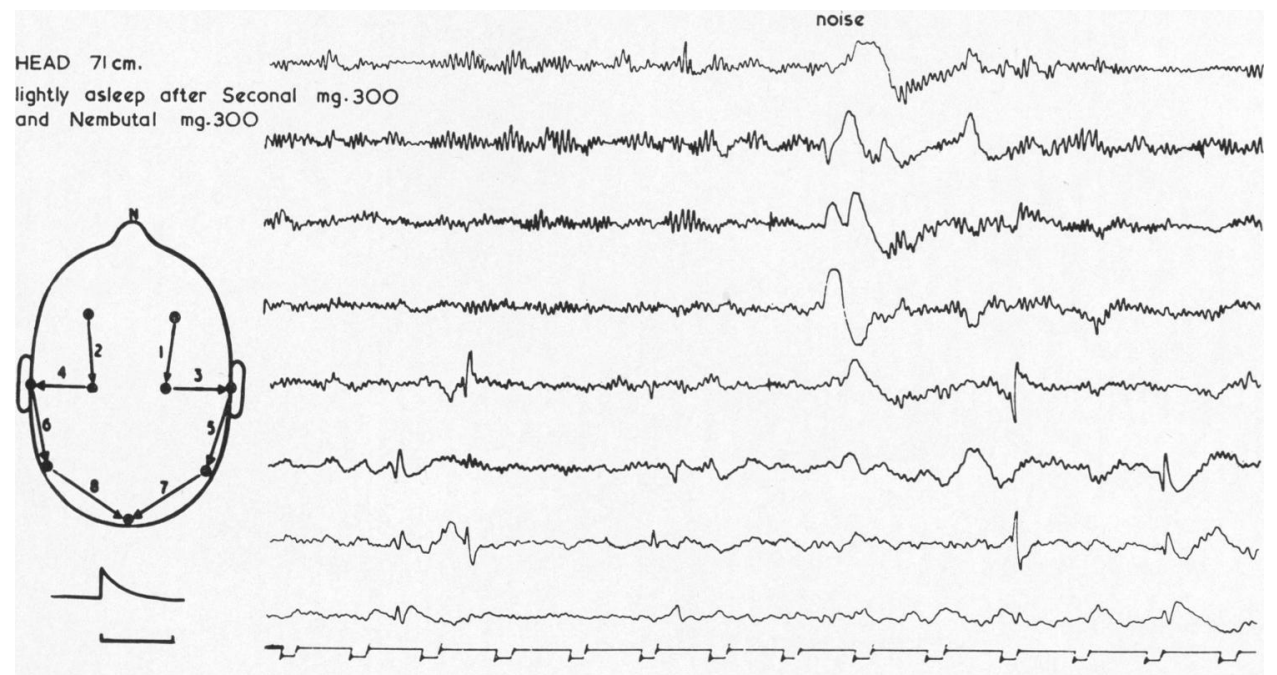

Fig. 5.-S.R., age 10 years and 6 months (head $71 \mathrm{~cm}$.). E.E.G. recorded on November 5, $1957(200 \mu \mathrm{v})$. Lightly asleep after seconal mg. 300 and nembutal mg. 300.

unsupported, and could speak only a few words. The hydrocephalus did not progress further, but she remained severely subnormal (I.Q. 40) and restless with occasional seizures. When she was admitted at the age of 10 years, it was difficult to approach her as she would bite and scratch most people. Air encephalography and a dye test demonstrated a communicating hydrocephalus due to basal cistern block. An E.E.G. (Fig. 5) could be taken only after the administration of a very large dose of barbiturates. Independent multifocal discharges were seen, maximal in the temporo-occipital regions. In spite of the very large size of the patient's head, which had reached $71 \mathrm{~cm}$., there was no marked asynchrony in the occurrence of sleep spindles (Fois et al., 1958). This girl is still in an institute for handicapped children, having apparently very infrequent seizures.

Other Aspects. From the clinical, and particularly from the E.E.G., aspect it was difficult to evaluate the importance of trauma and infection superimposed upon the hydrocephalus particularly when a number of therapeutic attempts had been carried out. A simple ventricular puncture through the fontanelle did not appear to induce gross alterations in the E.E.G. in two patients in whom continuous records were taken during slow withdrawal of C.S.F. In each case, two needles were inserted in the lateral ventricles near the lateral margins of the very wide anterior fontanelle. No spreading depression of the electrical activity was noticed after the insertion of the needles into the ventricles, either in these or in other patients. No marked changes in the E.E.G. were noticed when the intraventricular pressure had been lowered to about half its original value.

Case H.C. This baby was delivered by caesarean section because of disproportion, and was found to be hydrocephalic at birth. A ventriculogram at one month demonstrated a marked hydrocephalus due to a basal cistern block. The C.S.F. was xanthochromic with a raised protein content and under increased pressure. An E.E.G. taken at the age of 6 weeks showed asymmetry between the activities of the two hemispheres. Irregular slow activity and sharp elements were seen over the right hemisphere, while there was some reduction of activity over the anterior half of the left (Fig. 6). A marked bulging of the fontanelle could be seen when the baby cried. The asymmetry between the activities of the two hemispheres persisted in the E.E.G. in the following months. A ventriculo-peritoneal anastomosis at the age of $\mathbf{2}$ months was unsuccessful. A Spitz-Holter valve operation at the age of 5 months was not satisfactory. At the age of 7 months, continuous E.E.G.s were taken during slow withdrawal of C.S.F. The intraventricular pressure was reduced from 230 to $125 \mathrm{~mm}$. of C.S.F. over a period of 15 minutes (Fig. 7), and only minor E.E.G. changes occurred, probably related to the baby becoming very sleepy. The asymmetry noticed in the E.E.G. at the age of 6 weeks persisted, while the irregular slow activity and discharges over the right hemisphere had increased. The child died at the age of 9 months. Autopsy showed, in addition to a gross hydrocephalus due to a basal cistern block and a stenosis of the aqueduct, a dural sinus thrombosis and an extensive ventriculitis. The pallium was nowhere more than $1 \mathrm{~cm}$. in thickness.

In the E.E.G. marked asymmetry in the activity 


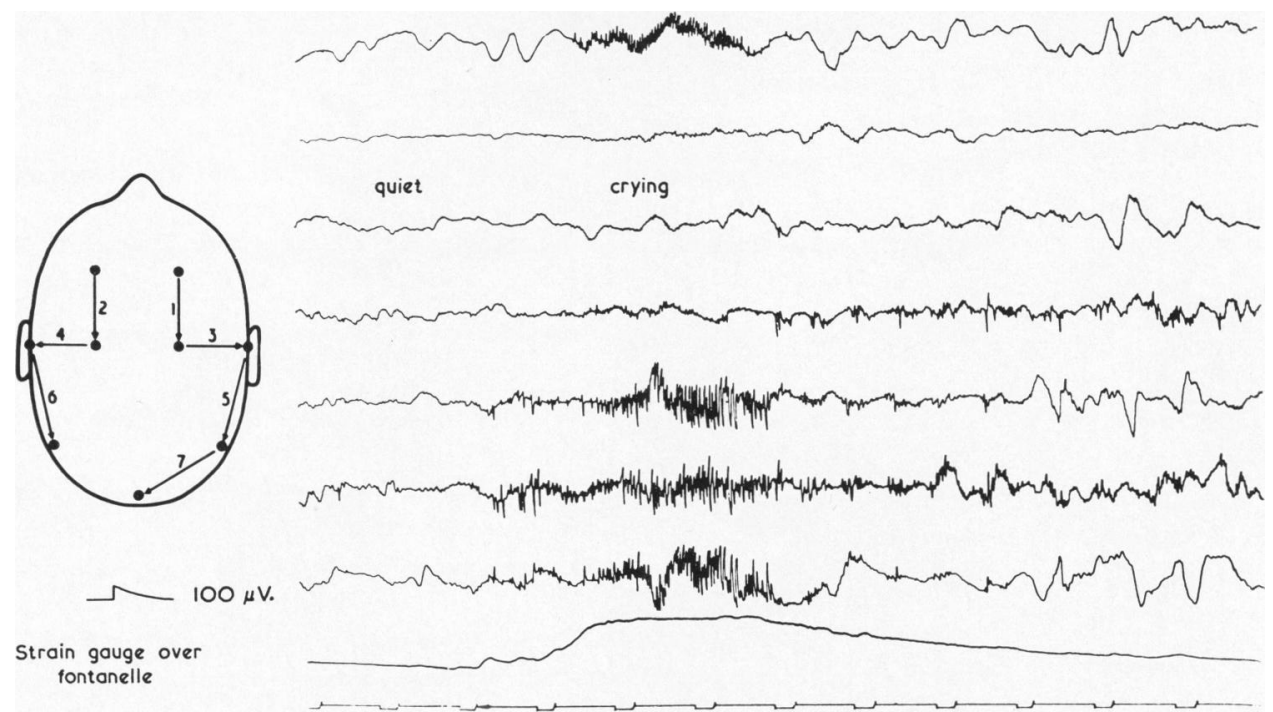

FIG. 6.-Helen C., age 6 weeks. E.E.G. on March 4, 1958.

Hydrocephalus.

of the two cerebral hemispheres was more commonly seen in cases of basal cistern block and aqueduct stenosis than in patients with Arnold-Chiari malformation, if we consider only the E.E.G.s taken before any therapeutic attempt.

Neither a simple Torkildsen operation, nor the insertion of a Forrest disc, substantially altered the E.E.G. features in the majority of patients. However, in three of the children where a disc had been inserted and both pre- and post-operative E.E.Gs. had been taken, an extensive flattening of the traces was found over the operated hemisphere a few weeks or months after the operation. In these cases, it seems probable that a large amount of fluid had been accumulating in the subdural space while the thin pallium collapsed extensively.

Case K.L. This boy was born after a 35-week pregnancy complicated by frequent vaginal bleeding. Some enlargement of the head was noticed after the age of 4 months. The baby was admitted to hospital, and dye and air studies demonstrated a severe generalized ventricular dilatation due to a basal cistern block. The first E.E.G. taken at 8 months showed a moderate abnormality over both fronto-central regions without constant asymmetries or paroxysmal features (Fig. 8). A Forrest disc operation was performed and the patient appeared to improve, but soon the hydrocephalus became progressive again. An E.E.G. taken at 18 months showed extensive flattening of the traces over the right hemisphere (Fig. 9), possibly related to a large subdural effusion. Subsequent ventriculo-peritoneal drainage proved unsuccessful, and the child died. Permission for a post-mortem examination could not be obtained.
In one patient, E.E.G.s were taken from the anterior frontal, sylvian, central and temporal regions before, during and after the introduction of a Spitz-Holter valve. The only appreciable change in the E.E.G. was some increase in slow activity over the right hemisphere, beginning a few seconds after the insertion of the catheter of the valve into the right lateral ventricle. There was no depression of the activity over that hemisphere in the following 15 minutes of continuous recording.

Poor visual acuity is believed to be common in patients with hydrocephalus. In a young child or baby, often with abnormal ocular movements, it may be difficult to assess whether vision is already lost. The difficulty in recognizing optic atrophy in children is well known, as pale discs are often seen in children with good vision. Attention, therefore, was directed towards the effect of rhythmic photic stimulation on the E.E.G. of our patients. None of the 35 children with recognizable E.E.G. responses to photic stimulation was eventually shown to be blind. In 12 further cases, no recognizable responses were elicited by rhythmic photic stimulation, and six of these were eventually considered to be completely blind. In the other six, some vision could be eventually demonstrated. When the responses to rhythmic photic stimulation were elicited, their amplitude was greater for low rates of flickering (two to four flashes per second) than for higher rates, this being a common feature in normal young children. In contrast with Gastaut's (1950) experience, in none of our patients 
did 'recruiting responses' to photic stimulation appear, and sharp waves at the vertex were seen in only two of our 50 patients.

\section{Conclusions}

From our studies on hydrocephalus in childhood, it appears that the E.E.G. features are related to the type, time and distribution of the lesions responsible for the development of hydrocephalus, rather than to the presence or severity of hydrocephaly. Neither slow nor rapid changes in the intraventricular pressure appeared to alter substantially the E.E.G. features in selected patients. There was no evidence that spreading depression phenomena might be started by a simple puncture of the brain and its covering, nor by the presence of two stainless steel needles in the lateral ventricles for periods of over half an hour, nor by the insertion of a SpitzHolter valve. Sharp waves at the vertex, considered as an important E.E.G. feature in hydrocephalus by other workers, were in fact uncommon in our material.

The presence of well-formed E.E.G. responses to photic stimulation suggested that vision was not totally lost in doubtful cases. After some operations on one lateral ventricle (Forrest disc), the

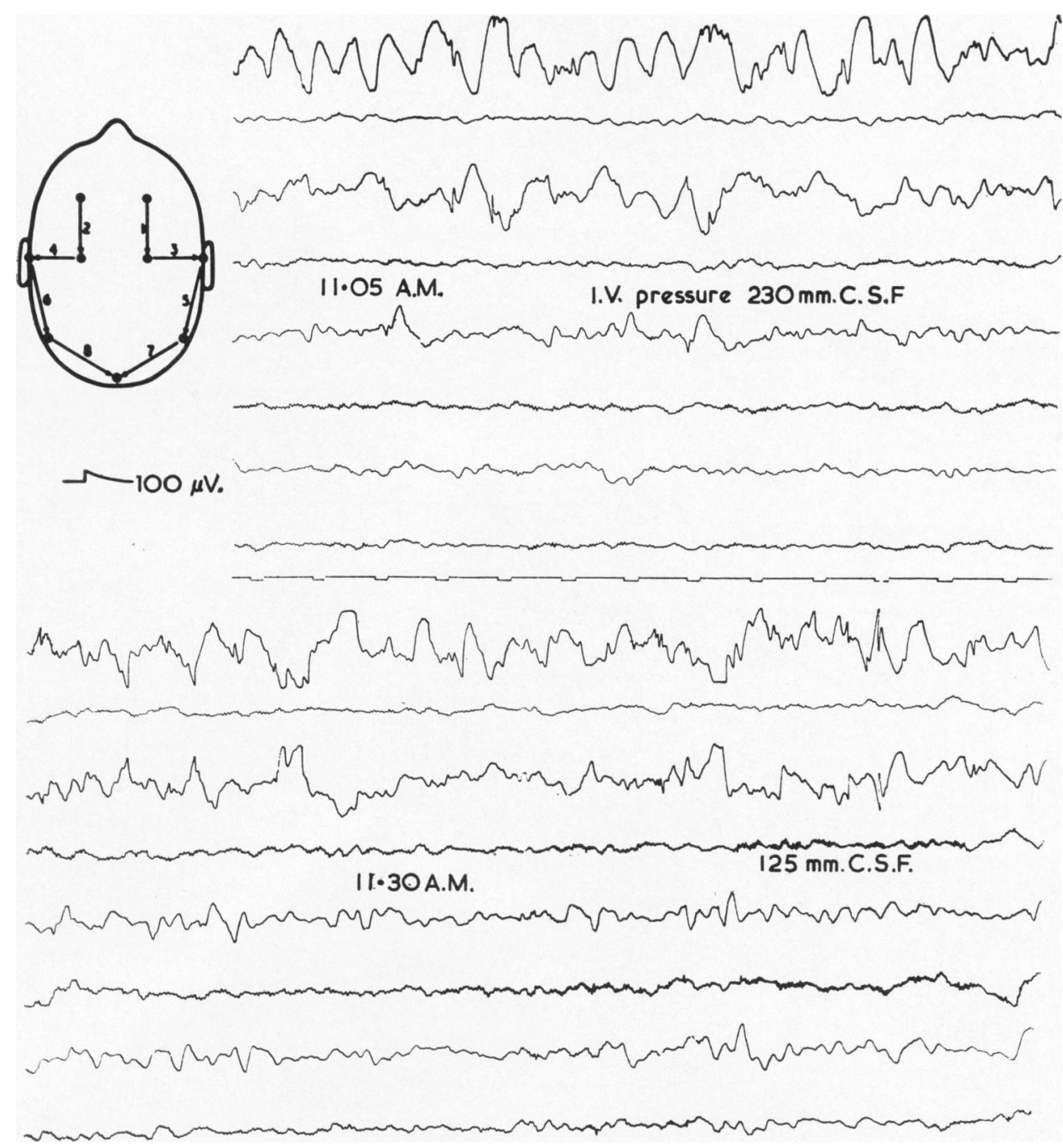

Fig. 7.-Helen C., age 7 months. E.E.G. on August 12, 1958 before and after slow withdrawal of C.S.F. (93 ml.) 


\section{thed $57 \mathrm{~cm}$}

BASAL C. BLOCK

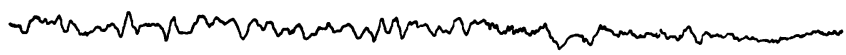

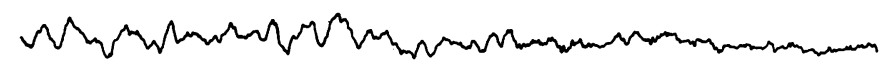

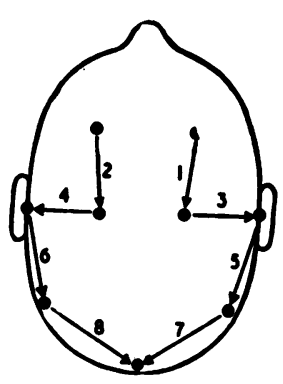

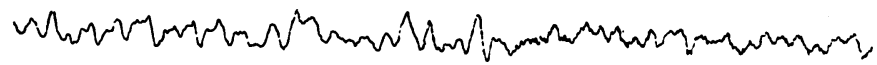

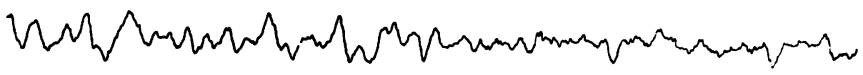

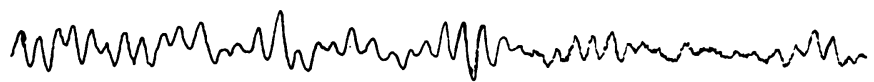

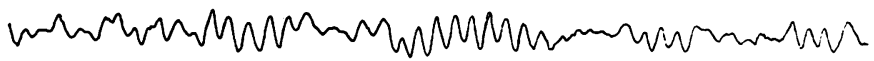

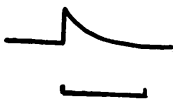

100

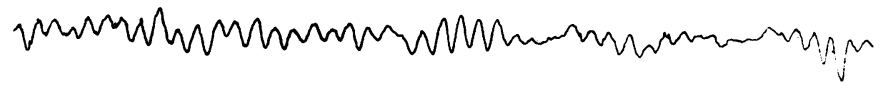

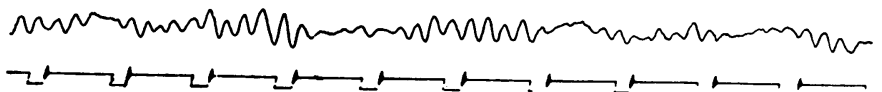

FIG. 8.-K.L., age 8 months (head $57 \mathrm{~cm}$.). E.E.G. recorded on May 20, 1957. Basal cistern block.

presence of a unilateral flattening of the E.E.G. was related either to the presence of a large local collection of fluid between the brain and the dura mater, or to an extensive neuronal loss. In general either single or serial E.E.G. investigations were of value in those differential diagnostic problems where information about the functional state of the brain would be unobtainable with other means.

BASAL C. BLOCK. Head $71 \mathrm{~cm}$.

Right Disc Operation 19.6.57.

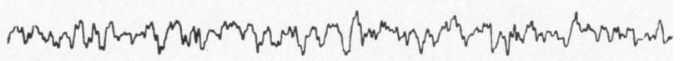

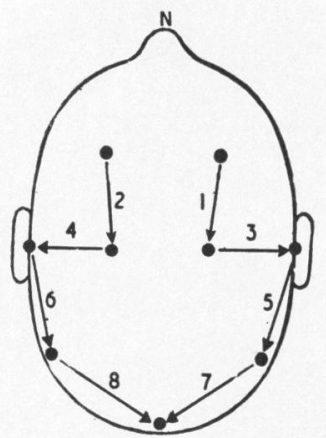

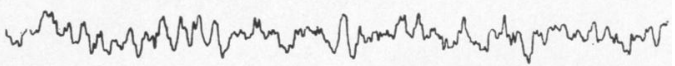

(1)

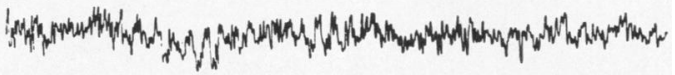

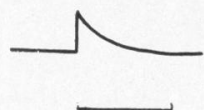

100

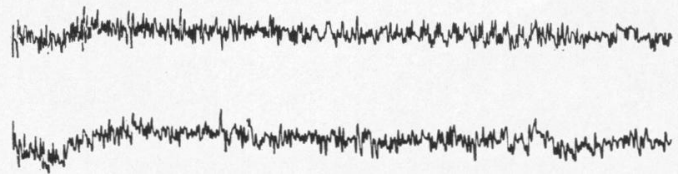

Fig. 9.-K.L., age 18 months (head $71 \mathrm{~cm}$.). E.E.G. February 14, 1958. Basal cistern block. Right disc operation on June 19 , 1957. 
Serial E.E.G. studies in our cases did not appear to help in the general prognostic outlook of selected cases.

As in other groups of patients, particularly children, the occurrence of discharges in the E.E.G. (focal spikes and sharp waves) was not limited to patients known to suffer from seizures.

It is a pleasure to acknowledge the help of our colleagues at The Hospital for Sick Children, Great Ormond Street, and at the Westminster Children's Hospital, and especially Dr. M. Bodian, Mr. G. H. Macnab and Mr. W. McKissock. One of us (K.M.L.) is indebted to the Research Committee of The Hospital for Sick Children for a Research Fellowship in Hydrocephalus and Spina Bifida.

\section{REFERENCES}

Cavazzuti, G. B., Canossi, G. C. and Bergonzini, R. (1957). L'idrocefalo infantile; aggiornamento clinico-etio-patogenetico; ricerche pneumoencefalografiche ed elettroencefalografiche; direttive del trattamento radiante. G. Psichiat. Neuropat., $85,827$.

Fois, A., Gibbs, E. L. and Gibbs, F. A. (1958). Bilaterally independent sleep patterns in hydrocephalus. A.M.A. Arch. Neurol. Psychiat., 79, 264.

Forrest, D. M., Laurence, K. M. and Macnab, G. H. (1957). Ventriculo-subdural drainage in infantile hydrocephalus; Analysis of early results. Lancet, 1, 1274 .

Gastaut, H. (1950). Un signe électroencéphalographique des hydrocéphalies: la réponse par recrutement au cours de la stimulation lumineuse intermittente. Rev. neurol., 82, 410.

Hill, J. D. N. and Parr, G. (1950). Electroencephalography.

Macdonald, London.
Laurence, K. M. (1957). The urinary phenolsulphonphthalein (phenol red) excretion test in hydrocephalus. Arch. Dis. Childh., 32, 413.

- (1959). Some applications of the urinary phenol-sulphonphthalein excretion test in hydrocephalus and related conditions. Brain, 82, 551.

Melchior J. C. (1961). Pneumoencephalography in Atrophic Brain Lesions in Infancy and Childhood. Frost-Hansen, Copenhagen. 